\title{
ON GENERALIZED COMMUTING ORDER OF AUTOMORPHISMS WITH QUASI-DISCRETE SPECTRUM
}

\author{
BY \\ NOBUO AOKI
}

0.0. Introduction. Abramov [1] has defined the notions of an automorphism of a finite measure space with quasi-discrete spectrum, using the concepts of quasiproper function and quasi-proper value introduced by Halmos and von Neumann [12]. For this class of quasi-proper functions Abramov defines an ascending sequence of abelian groups, which turns out to be a complete set of invariants for the classification of automorphisms with quasi-discrete spectrum. In addition he proves a representation theorem. In [11] an analogous theorem was proved for a homeomorphism of a compact space. Adler [2] has introduced the generalized commuting order of an automorphism on a finite measure space. The generalized commuting order is conjugacy invariant for automorphisms. [2] proves that the generalized commuting order of a totally ergodic translation of the measure space consisting of a compact metric abelian group is two. Furthermore, [5] gives conditions that every member of the generalized commuting order 2 have quasidiscrete spectrum.

In this paper we discuss the result first obtained by Abramov [1] in $\S 1$. In $\S 2$ we show a result stronger than the representation theorem of Abramov, Hahn and Parry. Our main result is to know an answer to the following question raised by Adler [2]. Let $T$ be an automorphism of a finite measure space, then are there automorphisms $T$ for $C N(T)=n$ for an integer $n$ including $C N(T)=\infty$ ? In $\S 3$, we mention a few examples concerning this question. Furthermore, for a totally ergodic automorphism of a finite measure space with quasi-discrete spectrum, we generalize the obtained examples.

I benefited from reading the papers by Adler [2], Hahn [10] and Hoare and Parry [14].

0. Preliminaries. By a dynamical system we mean a pair $(X, T)$ where $X$ is a compact Hausdorff space and $T$ is a homeomorphism of $X$ onto itself. We say that $(X, T)$ is minimal if $X$ contains no nonempty closed $T$-invariant set, and totally minimal if $\left(X, T^{m}\right)$ is minimal for any integer $m \neq 0$. Throughout, a homeomorphism $T$ is bicontinuous of $X$ onto itself. Let $C(X)$ be the Banach algebra of continuous complex valued functions on a compact Hausdorff space $X$. A homeomorphism $T$ induces an isometric isomorphism $V_{T}$ of the Banach algebra $C(X), V_{T} f(x)=f(T x)$.

Received by the editors June 10, 1969 and, in revised form, April 9, 1970.

Copyright (C) 1970, American Mathematical Society 
Let $(X, T)$ be totally minimal. We recall the following definition of quasi-proper function [10]. Let $G(T)_{0}$ be a group $\left\{\alpha \in K: V_{T} f(x)=\alpha f(x),|f(x)|=1\right.$ for $\left.f \in C(X)\right\}$ where $K$ is the unit circle in the complex plane. For $i>0$ let $G(T)_{i} \subset C(X)$ be the group of all functions $f$ such that $V_{T} f=g f,|f(x)|=1$ where $g \in G(T)_{i-1}$. We put $G(T)=\bigcup_{i>0}(T)_{i} .(X, T)$ is said to have quasi-discrete spectrum if $G(T)$ spans $C(X)$, and have discrete spectrum if $G(T)_{1}$ spans $C(X)$. If it ever happens that $G(T)_{n}=G(T)_{n+1}$, then $G(T)_{n}=G(T)_{n+k}$ for all $k$ and in this case we define $G N(T)$ $=\min \left\{n: G(T)_{n}=G(T)_{n+1}\right\}$ and otherwise $G N(T)=\infty$. It follows that $G(T)$ $=K \times O(T)$ where $O(T)$ is a subgroup of $G(T)$ isomorphic to the factor group $G(T) / K$ and the elements of $O(T)$ are linearly independent. If $(X, T)$ is totally minimal and has quasi-discrete spectrum, then there exists a unique $T$-invariant finite Borel measure [10]. Two compact Hausdorff spaces $X$ and $Y$ are homeomorphic if and only if their corresponding Banach algebras $C(X)$ and $C(Y)$ are isomorphic [9]. Whenever a compact Hausdorff space $X$ is metrizable, Halmos and von Neumann [12] have proved that if $(X, T)$ is minimal and $T$ an isometric homeomorphism on $X$ then it is possible to introduce into $X$ a multiplication so that $X$ becomes (with the original topology of $X$ ) a compact metric abelian group and $T$ becomes a translation. But a homeomorphism $T$ of the circle such that no power of $T$ has a fixed point is homeomorphic to a translation [8]. Hahn and Parry [11] have proved that if $(X, T)$ is totally minimal and has quasi-discrete spectrum then there exist a compact abelian group with the normalized Haar measure and a totally ergodic affine transformation $A$ on the space, and $T$ is homeomorphic to $A$. Let $X_{n}$ be the $n$-dimensional torus, i.e., $X_{n}=R^{n} / \sim$ where $R^{n}$ is the Euclidean plane and $\sim$ is the equivalence relation which identifies $n$-points in the plane if their corresponding coordinates differ by integers. A metric on $X_{n}$ can be defined in terms of the metric on $R^{n}$ by taking the distance between $n$-points of $X_{n}$ to be the minimal distance between any representatives of these points in $R^{n}$. The set of

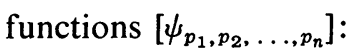

$$
\psi_{p_{1}, p_{2}, \ldots, p_{n}}\left(x_{1}, x_{2}, \ldots, x_{n}\right)=\exp \left[2 \pi i\left(p_{1} x_{1}+p_{2} x_{2}+\cdots+p_{n-1} x_{n-1}+p_{n} x_{n}\right)\right]
$$

where $p_{i}=0, \pm 1, \pm 2, \ldots$ and $i=1,2, \ldots, n$, forms a complete system of $C\left(X_{n}\right)$. The set of generators of a compact metric connected abelian group has a positive measure with respect to its Haar measure [13]. It is known that a translation $T_{r}: x \rightarrow x+r$ on a compact abelian group $X$ is ergodic if and only if $r$ is a generator of $X$. Let $(\Omega, \Sigma, \mu)$ be a finite measure space where $\Omega$ is a set of elements, $\Sigma$ a $\sigma$-field of measurable subsets of $X$, and $\mu$ a finite measure on $\Sigma$. We denote by $\Sigma(\mu)$ the Boolean $\sigma$-algebra by identifying sets in whose symmetric difference has zero measure, and $\mu$ is induced on the elements of $\Sigma(\mu)$ in the natural way. Let $L^{2}(\Sigma)$ be the Hilbert space of complex-valued square integrable functions defined on $(\Omega, \Sigma, \mu)$, but sometimes we use two symbols $L^{2}(\Omega)$ and $L^{2}(\Sigma(\mu))$ instead of $L^{2}(\Sigma)$. Let $T$ be an automorphism of $(\Omega, \Sigma, \mu)$ and we denote by $V_{T}: f(x) \rightarrow f(T x)$ $\left(f \in L^{2}(\Sigma)\right)$ the linear isometry induced by $T$. An automorphism of the measure 
algebra is called a metric automorphism. An automorphism $T$ of $(\Omega, \Sigma, \mu)$ induces a metric automorphism in the natural way and sometimes we denote by $T^{\prime}$ an induced metric automorphism. $T$ is said to be totally ergodic if $T^{n}$ is ergodic for every integer $n \neq 0$. We recall the following definition of quasi-proper function for a totally ergodic automorphism of $(\Omega, \Sigma, \mu)$ [1]. This definition is an analogue to that of a totally minimal dynamical system. Let $G_{\mu}(T)_{0}=\left\{\alpha \in K: V_{T} f=\alpha f\right.$ a.e., $\|f\|_{2}=1$ for $\left.f \in L^{2}(\Sigma)\right\}$, and for $i>0$ let $G_{\mu}(T)_{i} \subset L^{2}(\Sigma)$ be the set of all normalized functions $f$ such that $V_{T} f=g f$ a.e. where $g \in G_{\mu}(T)_{i-1}$. Then $G_{\mu}(T)_{i}$ is the set of quasi-proper functions of order $i . T$ is said to have quasi-discrete spetrum if $G_{\mu}(T)=\bigcup_{i>0} G_{\mu}(T)_{i}$ spans $L^{2}(\Sigma)$. Since $G_{\mu}(T)$ is a group, we follow that $G_{\mu}(T)$ $=K \times O_{\mu}(T)$ where $O_{\mu}(T)$ is a subgroup of $G_{\mu}(T)$. We denote by $G_{\mu} N(T)$ the least positive integer $n$ for which $G_{\mu}(T)_{n}=G_{\mu}(T)_{n+1}$ does happen and otherwise $G_{\mu} N(T)$ $=\infty$. Halmos and von Neumann [12] shows that a linear isometry $V$ on $L^{2}(\Sigma)$ onto itself is induced by an automorphism of the measure algebra if and only if both $V$ and $V^{-1}$ send every bounded function onto a bounded function and $V(f g)=V f \cdot V g$ whenever $f$ and $g$ are bounded functions. A necessary and sufficient condition that a closed subspace $H$ of $L^{2}(\Sigma)$ be of the form $H=L^{2}(\Phi(\mu))$ where $\Phi(\mu)$ is the smallest $\sigma$-algebra of $\Sigma(\mu)$ with respect to which all functions in $H$ are measurable is that $H$ contains a dense subalgebra consisting of bounded functions, constant functions and their complex conjugations [6]. If $G$ is any group, and $a$ any element of $G$, then we define subsets $C_{n}(a)(n=0,1,2, \ldots)$ of $G$ in the following way:

$$
\begin{aligned}
& C_{0}(a)=\{e\}, \\
& C_{n}(a)=\left\{b \in G: b a b^{-1} a^{-1} \in C_{n-1}(a)\right\} \quad(n=1,2, \ldots) .
\end{aligned}
$$

It is clear that $C_{n}(a) \subset C_{n+1}(a), n=0,1,2, \ldots$ The least $n$ for which $C_{n}(a)=C_{n+1}(a)$ is called the generalized commuting order of $a$ in $G$, and we denote by $C N(a)$ such an integer $n$. If $b a b^{-1} a^{-1}=a^{\prime}$ where $b, a, a^{\prime} \in G$ then it is clear that $C N(a)=C N\left(a^{\prime}\right)$. But the converse does not hold. Adler [2] has shown the following results: let $T_{a}$ be the translation by $a$ in a compact separable abelian group. If $T_{a}$ is totally ergodic, then $C N\left(T_{a}\right)=2$ and $C_{1}\left(T_{a}\right)$ is the group of translations, and $C_{2}\left(T_{a}\right)$ is the group consisting of translations composed with continuous group automorphisms; let $r$ be an irrational number of the 1-dimensional torus $X_{1}$, and let $\boldsymbol{n}$ be an integer, then for $T_{r, n}\left(x_{1}, x_{2}\right)=\left(x_{1}+r, x_{2}+n x_{1}\right)$ (additions $\left.\bmod 1\right), C N\left(T_{r, n}\right)=3$, and $C_{1}\left(T_{r, n}\right)$, $C_{2}\left(T_{r, n}\right)$ and $C_{3}\left(T_{r, n}\right)$ are groups. By Adler's ideas, [4] has proved, without the representation theorem due to Halmos and von Neumann, that the generalized commuting order of a totally ergodic metric automorphism with discrete spectrum on the measure algebra associated with a finite measure space is two.

1. Properties of automorphisms with quasi-discrete spectrum. If $X$ is a compact abelian group, $r \in X$ and $\beta$ is a continuous group automorphism of $X$, then $T(x)$ $=T_{r} \beta(x)$ is called an affine transformation of $X$ onto itself. For a totally ergodic, (with respect to the Haar measure) affine transformation on $X$, both definitions of 
the word "quasi-discrete spectrum," introduced by Abramov [1] and Hahn and Parry [11], coincide.

The next result was first obtained by Hahn and Parry [11].

LEMMA 1.1. Let $X$ be a compact connected abelian group with Haar measure on $X$. If a totally ergodic affine transformation $T(x)=T_{r} \beta(x), x \in X$, has quasi-discrete spectrum, then $(X, T)$ is a totally minimal dynamical system.

Proof. Since the totally ergodic affine transformation $T$ has quasi-discrete spectrum, we see that $O_{\mu}(T)(\mu$ is Haar measure on $X)$ is equal to the character group of $X$. Let $C_{n}(n=1,2, \ldots)$ be a set

$$
\left\{g: B^{n} g=1, g \text { a character of } X\right\}
$$

where $B$ is a homomorphism on the character group of $X$ defined by $B g=g^{-1} V_{\beta} g$. Then $\bigcup_{k=1}^{\infty} C_{k}$ is equal to the character group of $X$ if and only if $T$ has quasidiscrete spectrum [14]. Suppose that $x, y, z$ are in $X$. Suppose that $\left\{n_{j}: j \in \Delta\right\}$ is a net of integer such that

$$
\lim T^{n_{j}} x=\lim T^{n_{j} y}=z .
$$

Then $\lim g\left(T^{n_{j}} x\right)=\lim g\left(T^{n_{j}} y\right)=g(z)$ for every character $g$ of $X$. Here we prove by induction that if $g$ is a quasi-proper function belonging to $C_{n}$ for any integer $n$ then $g(x)=g(y)$. If $n=1$, then $g(x)=g(y)$ since $B g=1$ and

$$
\lim g\left(\beta^{n_{j}}\left(x y^{-1}\right)\right)=1 \text {. }
$$

Suppose now that all characters which are quasi-proper functions belonging to $C_{n}$ annihilate $x y^{-1}$. Let $g$ be a quasi-proper function of $C_{n+1}$. Then $B^{n+1} g=1$. Thus $B^{n}(B g)=1$ and $B g \in C_{n}$. Therefore $B g(x)=B g(y)$ and $g\left(\beta\left(x y^{-1}\right)\right)=g\left(x y^{-1}\right)$ which gives $g(x)=g(y)$. We have shown that the character group is equal to $\bigcup_{k=1}^{\infty} C_{k}$ and every character $g$ satisfies $g(x)=g(y)$. By the duality theorem, we have $x=y$. But this is a definition of distal. Let $N$ be the smallest $\beta$-invariant subgroup of the character group containing characters $f_{t_{1}}, f_{t_{2}}, \ldots, f_{t_{n}}$ and let ann $(N)$ be the annihilator of $N$. Then $X /$ ann $(N)$ is metrizable. If $T^{\prime}$ is the affine transformation on $X /$ ann $(N)$ induced by $T$, we see that $T^{\prime}$ is totally ergodic and distal. From ergodicity of $T^{\prime}$, there is an element $x^{\prime} \in X /$ ann $(N)$ such that $\left\{T^{\prime n} x^{\prime}: n=0, \pm 1, \pm 2, \ldots\right\}$ is dense in $X / \operatorname{ann}(N)$. Moreover, since $T^{\prime}$ is distal, $\left(X / \operatorname{ann}(N), T^{\prime}\right)$ is minimal [7]. This fact and connectedness of $X$ guarantee that $(X, T)$ is totally minimal.

The idea of the following theorem is essentially contained in Abramov [1].

THEOREM 1.2. Let $(\Omega, \Sigma, \mu)$ be a normalized measure space, and let $Q$ be a totally ergodic automorphism of $(\Omega, \Sigma, \mu)$ with quasi-discrete spectrum. Then there exist a compact connected abelian group $X$ with the normalized Haar measure and affine transformation $T(x)=T_{a} \beta(x), x \in X$, where $a \in X$ and $\beta$ is a continuous group automorphism of $X$, and $Q$ is conjugate to $T$. Furthermore, the dynamical system $(X, T)$ is totally minimal. If $(\Omega, \Sigma, \mu)$ is separable, then $X$ is metrizable. 
Proof. We denote by $X$ the character group of $O_{\mu}(Q)$ imposed by the discrete topology. If $(\Omega, \Sigma, \mu)$ is separable, $O_{\mu}(Q)$ is countable so that $X$ is metrizable. $X$ is a compact abelian group with the normalized Haar measure. Let $\langle\cdot, \cdot\rangle$ denote the pairing between $O_{\mu}(Q)$ and its dual. To define the linear isometry, we put

$$
V\left(\sum_{k=1}^{n} r_{k} f_{k}\right)=\sum_{k=1}^{n} r_{k}\left\langle\cdot, f_{k}\right\rangle, \quad f_{k} \in O_{\mu}(Q) .
$$

Then $V$ is an isometry which can be extended uniquely to an isometry of $L^{2}(\Sigma)$ onto $L^{2}(X)$. We suppose that $V$ is an extended linear isometry. Since $V$ satisfies the conditions of the multiplication theorem, there exists a metric isomorphism $\varphi$ such that $V=V_{\varphi}$. Now define $V^{\prime}$ on $L^{2}(X)$ by $V^{\prime}=V_{\varphi} V_{Q} V_{\varphi}^{-1}$ and put $O(Q)$ $=\left\{\langle\cdot, f\rangle: f \in O_{\mu}(Q)\right\}$. Then $V^{\prime}$ has quasi-discrete spectrum and $K \times O(Q)$ is invariant under $V^{\prime}$. Here we show that $V^{\prime}$ is an operator induced by an affine transformation on $X . V^{\prime}$ is an automorphism of $K \times O(Q)$ onto itself and a subgroup $K \times 1$ is mapped identically onto itself. We define maps

$$
P: O(Q) \rightarrow O(Q), \quad r: O(Q) \rightarrow K
$$

by $V^{\prime} g=r(g) P(g), g \in O(Q)$. We have $r(f g)=r(f) r(g)$ and $P(f g)=P(f) P(g)$ for $f, g \in O(Q)$. Therefore $r(\cdot)$ and $P(\cdot)$ are homomorphisms of $O(Q)$. To show that $P(\cdot)$ is one-to-one, let us put $P(f)=P(g)$ for $f, g \in O(Q)$, then we have $V^{\prime}\left(f g^{-1}\right)=$ $r\left(f g^{-1}\right)$ and $f g^{-1}=r\left(f g^{-1}\right) \in O(Q)$, i.e., $f(x)=g(x)$ for all $x \in X$. It is clear that $P(\cdot)$ is onto. We have shown that $P(\cdot)$ is an automorphism of $O(Q) . P(\cdot)$ therefore induces a continuous group automorphism $\beta$ of $X$. Since $r$ is a homomorphism of $O(Q)$ into $K, r$ is an element of $X$. Therefore

$$
V^{\prime} g(x)=r(g) P(g)=g(r) g(\beta x)=g\left(T_{r} \beta x\right)
$$

for all $x \in X$ and all $g$. We have proved that $V^{\prime}$ is an operator induced by $T_{r} \beta$, and $Q$ is conjugate to $T_{r} \beta$. Since $T_{r} \beta$ is totally ergodic and has quasi-discrete spectrum, it follows that $X$ is connected. It is clear from Lemma 1.1 that $(X, T)$ is totally minimal.

The next corollary is the result of Hahn and Parry [11] and Hoare and Parry [14].

COROLlaRY 1.3. Let $X$ be a compact connected abelian group with Haar measure on $X$. An ergodic affine transformation $T$ has quasi-discrete spectrum if and only if $(X, T)$ is totally minimal.

2. Behavior of affine transformations with quasi-discrete spectrum. We see that the continuous group automorphisms of $X_{n}$ are in correspondence with the invertible linear transformations of $R^{n}$ which preserve subset $Z_{n}$ of $R^{n}$ consisting of points with integer coordinates. Therefore if a fixed base is chosen in $X_{n}$, the automorphisms of $X_{n}$ are in one-to-one correspondence with $n \times n$ unimodular matrices. Let $\beta$ be a continuous group automorphism of $X_{n}$ and let [ $\left.\beta\right]$ denote the 
corresponding matrix. If $[\beta]=\left[a_{i j}: i, j=1,2, \ldots, n\right]$ then the automorphism $\beta$ is given by

$$
\beta\left(\left(x_{1}, x_{2}, \ldots, x_{n}\right)+Z_{n}\right)=\left(\left(\sum_{j=1}^{n} a_{1 j} x_{j}, \sum_{j=1}^{n} a_{2 j} x_{j}, \ldots, \sum_{j=1}^{n} a_{n j} x_{j}\right)+Z_{n}\right) .
$$

This equation is denoted by

$$
\beta\left(x_{1}, x_{2}, \ldots, x_{n}\right)=\left(\sum_{j=1}^{n} a_{1 j} x_{j}, \sum_{j=1}^{n} a_{2 j} x_{j}, \ldots, \sum_{j=1}^{n} a_{n j} x_{j}\right) \quad \text { (additions mod 1). }
$$

THEOREM 2.1. Let $T$ be a homeomorphism of $X_{n}$ onto itself. If a dynamical system $\left(X_{n}, T\right)$ is totally minimal and has quasi-discrete spectrum, then there is an affine transformation $T_{r} \beta$ of $X_{n}$ homeomorphic to $T$. Furthermore, $T_{r} \beta$ is homeomorphic some affine transformation given by some matrix

$$
\left[\begin{array}{ccccc}
1 & & & & \\
a_{21} & 1 & & 0 & \\
a_{31} & a_{32} & 1 & & \\
\ldots & & \ddots & \\
a_{n 1} & \ldots & a_{n n-1} & 1
\end{array}\right] \text { and } r^{\prime}=\left[\begin{array}{c}
r_{1}^{\prime} \\
r_{2}^{\prime} \\
\vdots \\
r_{n}^{\prime}
\end{array}\right] .
$$

In particular, if $a_{i j}=0$ for $i \neq j$ such that $2 \leqq i \leqq l$ and $1 \leqq j \leqq n$, then the numbers $r_{1}^{\prime}, r_{2}^{\prime}, \ldots, r_{l}^{\prime}$ are integrally independent.

Proof. Since $\left(X_{n}, T\right)$ is a totally minimal dynamical system with quasi-discrete spectrum, elements of $O(T)$ are linearly independent and $O(T)$ spans $C\left(X_{n}\right)$. We denote by $X$ the character group of $O(T)$ imposed by the discrete topology. $X$ is homeomorphic to $X_{n}$. Thus the rank of the character group $O(T)$ of $X$ is equal to the number $n$ since $X$ is the $n$-dimensional topological space. Since $X$ is connected and locally connected, $O(T)$ is the direct product of the free cyclic groups $C_{j}$, $j=1,2, \ldots, n$. Therefore $X$ is isomorphic to the $n$-dimensional torus $X_{n}$. If $T^{\prime}$ is the homeomorphism on $X$ induced by $T,\left(X, T^{\prime}\right)$ is a totally minimal dynamical system with quasi-discrete spectrum. Since $X$ is the $n$-dimensional torus, we may suppose that $X=X_{n}$. Then

$$
V_{T^{\prime}}\left(K \times\left[\psi_{p_{1}, p_{2}, \ldots, p_{n}}\right]\right)=\left(K \times\left[\psi_{p_{1}, p_{2}, \ldots, p_{n}}\right]\right) .
$$

As we did in Theorem 1.2, we follow that $T^{\prime}$ is an affine transformation $T_{r} \beta$ such that $T_{r}$ is a translation of $X_{n}$ and $\beta$ a continuous group automorphism of $X_{n}$. Thus $G\left(T_{r} \beta\right)=K \times\left[\psi_{p_{1}, p_{2}}, \ldots, p_{n}\right]$ and $G N\left(T_{r} \beta\right)$ is finite. If $G N\left(T_{r} \beta\right)=m$ and

$$
G\left(T_{r} \beta\right)_{i}=\left[\psi_{p_{1}, p_{2}, \ldots, p_{l_{i}}}\right], \quad i=1,2, \ldots, m,
$$

we choose a base dependent on (1) in $X_{n}$. For the base in $X_{n}$, there exists some unimodular matrix

$$
\left[\beta^{\prime}\right]=\left[a_{i j}: i, j=1,2, \ldots, n\right]
$$


so that the continuous group automorphism given by the matrix [ $\left.\beta^{\prime}\right]$ is isomorphic to $\beta$. Thus $T_{r} \beta$ is homeomorphic to the affine transformation $T_{r} \beta^{\prime}$ where

$$
r^{\prime}=\left[\begin{array}{c}
r_{1}^{\prime} \\
\vdots \\
r_{n}^{\prime}
\end{array}\right]
$$

Since the operator $V_{\beta^{\prime}}$ is identical on $\left[\psi_{p_{1}, p_{2}, \ldots, p_{l_{1}}}\right]$, it follows that

$$
\left[\beta^{\prime}\right]=\left[\begin{array}{c:c}
E_{1} & 0 \\
\hdashline * & \multicolumn{2}{c}{*}
\end{array}\right]
$$

where $E_{1}$ is the identity matrix of order $l_{1} \times l_{1}$. For every $g \in\left[\psi_{p_{1}, p_{2}, \ldots, p_{l_{2}}}\right]$,

where

$$
V_{\beta^{\prime}} g\left(x_{1}, x_{2}, \ldots, x_{l_{2}}\right)=g^{\prime}\left(x_{1}, x_{2}, \ldots, x_{n}\right) g\left(x_{1}, x_{2}, \ldots, x_{l_{2}}\right)
$$

$$
\begin{aligned}
g^{\prime}\left(x_{1}, x_{2}, \ldots, x_{n}\right) & \\
= & \exp \left[2 \pi i\left(\left(\sum_{k=1}^{l_{2}} p_{k} a_{k 1}\right) x_{1}+\left(\sum_{k=1}^{l_{2}} p_{k} a_{k 2}\right) x_{2}+\cdots+\left(\sum_{k=1}^{l_{2}} p_{k} a_{k n}\right) x_{n}\right)\right] \\
& \quad \exp \left[-2 \pi i\left(p_{1} x_{1}+p_{2} x_{2}+\cdots+p_{l_{1}} x_{l_{1}}\right)\right],
\end{aligned}
$$

and $g^{\prime}$ is an element of $G\left(T_{r}, \beta^{\prime}\right)=\left[\psi_{p_{1}, p_{2}, \ldots, p_{l_{1}}}\right]$. From this fact, the form of $\left[\beta^{\prime}\right]$ is the following matrix

$$
\left[\beta^{\prime}\right]=\left[\begin{array}{c:cc}
E_{1} & & 0 \\
\hdashline & E_{2} & \\
\hdashline & * &
\end{array}\right]
$$

where $E_{1}$ is the identity matrix of order $l_{1} \times l_{1}$ and $E_{2}$ the identity matrix of order $\left(l_{2}-l_{1}\right) \times\left(l_{2}-l_{1}\right)$. From such an argument we see that the form of $\left[\beta^{\prime}\right]$ is the following triangular matrix

$$
\left[\beta^{\prime}\right]=\left[\begin{array}{c:cccc}
E_{1} & & & & \\
& E_{2} & & & 0 \\
& & E_{3} & & \\
& & & & \\
& * & & \ddots & \\
& & & & E_{m}
\end{array}\right]
$$


where $E_{j}$ is the identity matrix of order $\left(l_{j}-l_{j-1}\right) \times\left(l_{j}-l_{j-1}\right)$ (but $\left.l_{0}=0\right)$ for $j=1,2, \ldots, m$. The fact that $r_{1}^{\prime}, r_{2}^{\prime}, \ldots, r_{l_{1}}^{\prime}$, are integrally independent follows immediately from the fact that $\left(X, T_{r}, \beta^{\prime}\right)$ is minimal.

It is well known that on the 1-dimensional torus $X_{1}$ there exist only two continuous group automorphisms, the identical automorphism and another automorphism $\beta$ for which $\beta x=-x, x \in X_{1}$.

We have the next corollary here.

COROLlARY 2.2. Let $T$ be a homeomorphism of $X_{1}$ onto itself and if a dynamical system $\left(X_{1}, T\right)$ is totally minimal and has quasi-discrete spectrum, then there is a translation of $X_{1}$ homeomorphic to $T$.

Let $T$ be a homeomorphism of the 2-dimensional torus $X_{2}$, and defined by

$$
\left.T:\left(x_{1}, x_{2}\right) \rightarrow\left(x_{1}+r, x_{2}+n x_{1}\right) \quad \text { (additions } \bmod 1\right)
$$

where $r$ is a real number and $n$ an integer. Such a transformation is called a skew product transformation of $X_{2}$ [3].

COROLlaRY 2.3. If $\left(X_{2}, T\right)$ is totally minimal and has quasi-discrete spectrum, then there is a skew product transformation of $X_{2}$ homeomorphic to $T$.

This is direct from Theorem 2.1.

COROLlary 2.4. Let $\left(X_{n}, T\right)$ be a totally minimal dynamical system with quasidiscrete spectrum and let $C N(T)=2$. Then there is a following affine transformation $T_{r} \beta$ homeomorphic to $T$,

$T_{r} \beta:\left(x_{1}, x_{2}, \ldots, x_{n}\right)$

$$
\rightarrow\left(x_{1}+r_{1}, \ldots, x_{l}+r_{l}, x_{l+1}+\sum_{j=1}^{l} a_{l+1 j} x_{j}+r_{l+1}, \ldots, x_{n}+\sum_{j=1}^{l} a_{n j} x_{j}+r_{n}\right)
$$

(additions mod 1)

where each $a_{\iota j}$ is some integer and each $r_{j}$ some real number, and moreover the numbers $r_{1}, r_{2}, \ldots, r_{l}$ are integrally independent.

COROLlaRY 2.5. Let $\left(X_{n}, T\right)$ be a totally minimal dynamical system with quasidiscrete spectrum and let $G N(T)=n$. Then there is a following affine transformation $T_{r} \beta$ homeomorphic to $T$,

$$
\begin{array}{r}
T_{r} \beta:\left(x_{1}, x_{2}, \ldots, x_{n}\right) \\
\rightarrow\left(x_{1}+r_{1}, x_{2}+a_{21} x_{1}+r_{2}, \ldots, x_{n-1}+\sum_{j=1}^{n-2} a_{n-1} x_{j}+r_{n-1}, x_{n}+\sum_{j=1}^{n-1} a_{n j} x_{j}+r_{n}\right) \\
\quad(\text { additions mod 1) }
\end{array}
$$

where each $a_{i j}$ is some integer, but $a_{j-1}$ is nonzero for $j=2, \ldots, n$, and each $r_{j}$ some real number, but $r_{1}$ irrational. 
Proof. From Theorem 2.1, $T$ is homeomorphic to some affine transformation $T_{r} \beta$ such that the matrix $[\beta]$ is of the following form

$$
[\beta]=\left[\begin{array}{ccccc}
1 & & & & \\
a_{21} & 1 & & & 0 \\
a_{31} & a_{32} & 1 & & \\
\cdots & & & \ddots & \\
a_{n 1} & & \cdots & a_{n n-1} & 1
\end{array}\right] \text { and } r=\left[\begin{array}{c}
r_{1} \\
r_{2} \\
\vdots \\
r_{n}
\end{array}\right]
$$

where each number $a_{i j}$ is some integer and each number $r_{j}$ some real. But $a_{j-1}$ is nonzero for $j=2, \ldots, n$ since $G N(T)=n$ and from (totally) minimality of $\left(X_{n}, T_{r} \beta\right), r_{1}$ is irrational.

COROllaRY 2.6. Let $T$ be a totally ergodic automorphism of a finite measure space $(\Omega, \Sigma, \mu)$ with quasi-discrete spectrum. Then there exist metric automorphisms $W$ and $S$ such that $W$ has each function of $O_{\mu}(T)$ as a proper function and $V_{S}$ maps $O_{\mu}(T)$ onto itself, and the metric automorphism of $T$ is equal to $S W$.

The proof of Corollary 2.6 is similar to [5].

3. Generalized commuting order of transformations. As pointed out in Adler [2], it is interesting to know an answer to the following question: are there examples for $C N(T)=n$ for an integer $n$ including $C N(T)=\infty$ ? The next example shows that this question has a positive answer.

THEOREM 3.1. Let $T$ be an affine transformation of $X_{n}$ and let $\left(X_{n}, T\right)$ be a totally minimal dynamical system (with quasi-discrete spectrum). If $G N(T)=n$, then $C N(T)=n+1$.

Proof. From Theorem 2.1 we may suppose that the affine transformation $T$ is written as follows

$$
\begin{aligned}
T:\left(x_{1},\right. & \left.x_{2}, \ldots, x_{n}\right) \\
& \rightarrow\left(x_{1}+r_{1}, x_{2}+a_{21} x_{1}+r_{2}, \ldots, x_{n-1}+\sum_{j=1}^{n-2} a_{n-1 j} x_{j}+r_{n-1}, x_{n}+\sum_{j=1}^{n-1} a_{n j} x_{j}+r_{n}\right)
\end{aligned}
$$

(additions mod 1)

where each number $a_{i j}$ is an integer and $r_{j}, j=1,2, \ldots, n$, are real. Since $G N(T)=n$, from Corollary 2.5 , the integer $a_{j-1}$ is nonzero for $j=2, \ldots, n$ and $r_{1}$ irrational. We show by induction that members of $C_{n}(T), n=1,2, \ldots$, are affine transformations. If $n=1$, then for $S_{1} \in C_{1}(T)$ we have $S_{1} T=T S_{1}$ and therefore

$$
V_{S_{1}}\left(K \times\left[\psi_{p_{1}, p_{2}, \ldots, p_{n}}\right]\right)=K \times\left[\psi_{p_{1}, p_{2}, \ldots, p_{n}}\right] .
$$

From this relation and the proof of Theorem 1.2, we see that $S_{1}$ is an affine 
transformation. Let members of $C_{n}(T)$ be affine transformations and let $S_{n+1} \in C_{n+1}(T)$. Then $S_{n+1} T S_{n+1}^{-1}=S_{n} T$ where $S_{n} \in C_{n}(T)$. $T$ and $S_{n} T$ are affine transformations, and $\left(X_{n}, T\right)$ and $\left(X_{n}, S_{n} T\right)$ are totally minimal dynamical systems (with quasi-discrete spectrum). Therefore it follows that

$$
V_{S_{n+1}}\left(K \times\left[\psi_{p_{1}, p_{2}, \ldots, p_{n}}\right]\right)=K \times\left[\psi_{p_{1}, p_{2}, \ldots, p_{n}}\right] .
$$

We see easily that $S_{n+1}$ is an affine transformation. We have shown that the members of $\bigcup_{n=0}^{\infty} C_{n}(T)$ are affine transformations. If $S_{n+2} \in C_{n+2}(T)$ then we can write $S_{n+2} T S_{n+2}^{-1}=S_{n+1} T$ for some $S_{n+1} \in C_{n+1}(T)$. Since $S_{n+1} T$ is homeomorphic to $T,\left(X_{n}, S_{n+1} T\right)$ is totally minimal and $G N\left(S_{n+1} T\right)=n$. The affine transformation $S_{n+1}$ has a representation as follows:

$$
S_{n+1}=T_{r_{n+1}} \beta_{n+1}
$$

where $T_{r_{n+1}}$ is a translation of $X_{n}$ and $\beta_{r+1}$ is a continuous group automorphism of $X_{n}$. We put $T=T_{r} \beta$ for convenience. Since $S_{n+1} T$ is homeomorphic to $T$ and since

$$
S_{n+1} T=T_{r_{n+1}+\beta_{n+1}(r)} \beta_{n+1} \beta
$$

has quasi-discrete spectrum and $G N\left(S_{n+1} T\right)=n$, we see by induction that the matrix $\left[\beta_{n+2}\right]$ is lower triangular. $\left[\beta_{n+1} \beta\right]$ is a lower triangular matrix such that the numbers 1 appear throughout the diagonal, because the spectrum of $S_{n+1} T$ is quasi-discrete. Thus we follow that $\left[\beta_{n+1}\right]$ is a matrix such that

$$
\left[\begin{array}{cccc}
1 & & & \\
& 1 & & 0 \\
& & \ddots & \\
& * & & \\
& & & 1
\end{array}\right] .
$$

We show now that $C_{n+1}(T)=C_{n+2}(T)$. Let $S_{n+2} \in C_{n+2}(T)$. Then $S_{n+2} T S_{n+2}^{-1} T^{-1}$ $=S_{n+1}$ where $S_{n+1} \in C_{n+1}(T)$. Furthermore, for the affine transformation $S_{n+1}$ $=T_{r_{n+1}} \beta_{n+1}$,

$$
S_{n+1} T S_{n+1}^{-1} T^{-1}=S_{n}
$$

where $S_{n} \in C_{n}(T)$ and $S_{n}=T_{r_{n}} \beta_{n}$. Then from (1),

$$
\begin{aligned}
\beta_{n+1} \beta & =\beta_{n} \beta \beta_{n+1}, \\
r_{n+1}+\beta_{n+1}(r) & =r_{n}+\beta_{n}(r)+\beta_{n} \beta\left(r_{n+1}\right) .
\end{aligned}
$$

From the fact that the spectrum of $S_{n} T$ is quasi-discrete, the matrix $\left[\beta_{n} \beta\right]$ is a lower triangular matrix such that the numbers 1 appear throughout the diagonal. 
Furthermore, since the numbers 1 appear throughout the diagonal of $\left[\beta_{n+1}\right]$ and from the relation (2), we see that

$$
\left[\beta_{n}\right]=\left[\begin{array}{cccccc}
1 & & & & \\
0 & & & & \\
b_{31} & 0 & 1 & & 0 & \\
\vdots & \ddots & \ddots & \ddots & \\
b_{n 1} & \cdots & b_{n n-2} & 0 & 1
\end{array}\right]
$$

where each $b_{i j}$ is some integer. Therefore, from the relations (3) and (4), it is easily to see that

$$
r_{n}=\left[\begin{array}{l}
0 \\
r_{n 2} \\
\vdots \\
r_{n n}
\end{array}\right] .
$$

Next, for the affine transformation $S_{n}=T_{r_{n}} \beta_{n}$, we have $S_{n} T S_{n}^{-1}=S_{n-1} T$ where $S_{n-1} \in C_{n-1}(T)$, and therefore $S_{n-1}$ is equal to an affine transformation $T_{r_{n-1}} \beta_{n-1}$ such that $\beta_{n-1}$ has a unimodular matrix

$$
\left[\beta_{n-1}\right]=\left[\begin{array}{cccccc}
1 & & & & & \\
0 & 1 & & & & \\
0 & 0 & 1 & & 0 & \\
c_{41} & 0 & 0 & 1 & & \\
\vdots & \ddots & \ddots & \ddots & \ddots & \\
c_{n 1} & \cdots & c_{n n-3} & 0 & 0 & 1
\end{array}\right] \text { and } r_{n-1}=\left[\begin{array}{l}
0 \\
0 \\
r_{n-13} \\
\vdots \\
r_{n-1}
\end{array}\right]
$$

Following this argument step-by-step, we get that $S_{3} T S_{3}^{-1}=S_{2} T$ where $S_{2} \in C_{2}(T)$, and the behavior of $S_{2}$ is the following

$$
S_{2}\left(x_{1}, x_{2}, \ldots, x_{n}\right)=\left(x_{1}, x_{2}, \ldots, x_{n-1}, x_{n}+r_{2 n}\right) \quad(\text { addition mod 1) }
$$

where $r_{2 n}$ is some real number. Therefore it follows that $S_{2}$ commutes with $T$, i.e., $S_{2} \in C_{1}(T)$. From this fact,

$$
S_{3} \in C_{2}(T), \ldots, S_{n-1} \in C_{n-2}(T), S_{n} \in C_{n-1}(T)
$$

and, from $S_{n+1} T S_{n+1}^{-1} T^{-1}=S_{n}$,

$$
S_{n+1} \in C_{n}(T) \text { and } S_{n+2} \in C_{n+1}(T) .
$$

Thus we have shown that $C_{n+1}(T)=C_{n+2}(T)$.

We give a translation $T_{d}$ :

$$
\left(x_{1}, x_{2}, \ldots, x_{n}\right) \rightarrow\left(x_{1}+d_{1}, x_{2}+d_{2}, \ldots, x_{n}+d_{n}\right) \quad \text { (additions mod 1) }
$$


where $d_{1}, d_{2}, \ldots, d_{n-1}$ and $d_{n}$ are nonzero real numbers, but not integers. Then we have the following equation

$$
\begin{aligned}
& T_{d} T T_{d}^{-1} T^{-1}\left(x_{1}, x_{2}, \ldots, x_{n}\right) \\
& \quad=\left(x_{1}, x_{2}+d_{2}^{(2)}, x_{3}+d_{3}^{(2)}, \ldots, x_{n}+d_{n}^{(2)}\right) \quad \text { (additions mod 1) }
\end{aligned}
$$

where

$$
d_{k}^{(2)}=-\sum_{j=1}^{k-1} a_{k j} d_{j}, \quad k=2,3, \ldots, n
$$

Putting

$$
T_{d^{(2)}}\left(x_{1}, x_{2}, \ldots, x_{n}\right)=\left(x_{1}, x_{2}+d_{2}^{(2)}, x_{3}+d_{3}^{(2)}, \ldots, x_{n}+d_{n}^{(2)}\right) \quad(\text { additions mod 1), }
$$

we have that

$$
T_{d^{(2)}} T T_{d^{(2)}}^{-1} T^{-1}\left(x_{1}, x_{2}, \ldots, x_{n}\right)=\left(x_{1}, x_{2}, x_{3}+d_{3}^{(3)}, x_{4}+d_{4}^{(3)}, \ldots, x_{n}+d_{n}^{(3)}\right)
$$

(additions mod 1)

where

$$
d_{k}^{(3)}=-\sum_{j=2}^{k-1} a_{k j} d_{j}^{(2)}, \quad k=3,4, \ldots, n .
$$

We obtain from the argument that

$$
T_{d^{(n)}} T\left(x_{1}, x_{2}, \ldots, x_{n}\right)=T T_{d^{(n)}}\left(x_{1}, x_{2}, \ldots, x_{n}\right)
$$

where $d_{n}^{(n)}=-a_{n-1} d_{n-1}^{(n-1)}$ and

$$
T_{d^{(n)}}\left(x_{1}, x_{2}, \ldots, x_{n}\right)=\left(x_{1}, x_{2}, \ldots, x_{n}+d_{n}^{(n)}\right) \quad \text { (addition mod 1). }
$$

Since $a_{j-1}$ is a nonzero integer for $j=2, \ldots, n$, we can choose nonzero real numbers (but not integers) $d_{1}, d_{2}, \ldots, d_{n-1}$ and $d_{n}$ such that

$$
\begin{array}{rc}
d_{k}^{(2)} \neq 0 & \text { for } k=2,3,4, \ldots, n, \\
d_{k}^{(3)} \neq 0 & \text { for } k=3,4,5, \ldots, n, \\
d_{k}^{(4)} \neq 0 & \text { for } k=4,5,6, \ldots, n, \\
d_{k}^{(n-1)} \neq 0 & \text { for } k=n-1, n, \\
d_{n}^{(n)} \neq 0 . &
\end{array}
$$

In particular, we can choose $d_{1}$ such that the number $-\frac{1}{2} \cdot d_{1}$ is the first coordinate $r_{1}$ of $r=\left(r_{1}, r_{2}, \ldots, r_{n}\right)$. For such real numbers $d_{j}, j=1,2, \ldots, n$, and the translation $T_{d}$ where $d=\left(d_{1}, d_{2}, \ldots, d_{n}\right)$, it follows that $T_{d^{(n)}} T=T T_{d^{(n)}}$, and since $d_{n}^{(n)} \neq 0$ $(\bmod 1), T_{d^{(n)}}$ is not the identical map. Thus we have that $T_{d} \in C_{n}(T)-C_{n-1}(T)$. Here we put $S=T_{b} \beta^{\prime}$ where $\beta^{\prime}$ is a group automorphism of $X_{n}$ such that $\beta^{\prime} x=-x$, $x \in X_{n}$, and $T_{b}$ is a translation determined by the element $b$ satisfying the equation $b+\left[\beta^{\prime}\right][r]=d+r+[\beta][b]$. Then $S T S^{-1} T^{-1}=T_{d}$ and therefore $S \in C_{n+1}(T)-C_{n}(T)$. We have shown that $C N(T)=n+1$. 
COROLlary 3.2. Let $T$ be an affine transformation and let $\left(X_{1}, T\right)$ be a totally minimal dynamical system (with quasi-discrete spectrum). If $G N(T)=1$, then $C N(T)=2$.

The corollary was shown by Adler [2].

Corollary 3.3. Let $T$ be a skew product transformation of $X_{2}$ and let $\left(X_{2}, T\right)$ be a totally minimal dynamical system (with quasi-discrete spectrum) and let $G N(T)$ $=2$. Then $C N(T)=3$.

The statement is direct from Theorem 3.1.

The following theorem is a result better than Theorem 3.1 .

THEOREM 3.4. Let $T$ be an affine transformation of $X_{n}$ and let $\left(X_{n}, T\right)$ be a totally minimal dynamical system (with quasi-discrete spectrum) with $G N(T)=m$. Then $C N(T)=m+1$.

Proof. We put $T=T_{r} \beta$ where $T_{r}$ is a translation of $X_{n}$ and $\beta$ a continuous group automorphism of $X_{n}$. From Theorem 2.1 and the analogous argument of Corollary 2.5, we may suppose that since $G N(T)=m$, the affine transformation $T$ is written as follows

$$
\begin{aligned}
& T_{r} \beta:\left(x_{1}, x_{2}, \ldots, x_{n}\right) \\
& \rightarrow\left(x_{1}+r_{1}, \ldots, x_{l_{1}}+r_{l_{1}}, x_{l_{1}+1}+\sum_{j=1}^{l_{1}} a_{l_{1}+1} x_{j}+r_{l_{1}+1}, \ldots,\right. \\
& x_{l_{2}}+\sum_{j=1}^{l_{1}} a_{l_{2} j} x_{j}+r_{l_{2}}, \ldots, x_{l_{m-1}+1} \\
& \left.+\sum_{j=1}^{l_{m-1}} a_{l_{m-1}+1} x_{j}+r_{l_{m-1}+1}, \ldots, x_{l_{m}}+\sum_{j=1}^{l_{m-1}} a_{l_{m} j} x_{j}+r_{l_{m}}\right) \\
& \quad(\text { additions mod } 1) .
\end{aligned}
$$

Here each $a_{i j}$ is an integer and each $l_{j}$ an integer such that $l_{m}=n$ and there is at the least one nonzero integer in

$$
\left\{a_{l_{i-1}+1 l_{i-2}+1}, a_{l_{i-1}+1 l_{i-2}+2}, \ldots, a_{l_{i-1}+1 l_{i-1}}\right\} \quad\left(l_{0}=0\right)
$$

for $i=2,3, \ldots, m$. Since $T$ is totally ergodic, the real numbers $r_{k}, k=1,2, \ldots, l_{1}$, are integrally independent. Thus the matrix $[\beta]$ is of the form

$$
\left[\begin{array}{ccccc}
E_{11} & & & & \\
A_{21} & E_{22} & & & 0 \\
A_{31} & A_{32} & E_{33} & & \\
& \ldots & & \ddots & \\
A_{m 1} & \ldots & A_{m m-1} & E_{m m}
\end{array}\right]
$$

where $E_{i i}, i=1,2, \ldots, m$, are the identity matrices of order $\left(l_{i}-l_{i-1}\right) \times\left(l_{i}-l_{i-1}\right)$ 
and $A_{i j}, i \neq j, i=2,3, \ldots, m, j=1,2, \ldots, m$, are matrices of order $\left(l_{i}-l_{i-1}\right) \times$ $\left(l_{j}-l_{j-1}\right)$ (but $l_{0}=0$ ). Since $T$ has quasi-discrete spectrum and $G N(T)=m$, the blocks of $[\beta], A_{i-1}, i=2,3, \ldots, m$, are nonzero matrices and there is at the least one nonzero integer in the first row

$$
\left(a_{l_{i-1}+1 l_{i-2}+1}, a_{l_{i-1}+1 l_{i-2}+2}, \ldots, a_{l_{i-1}+1 l_{i-1}}\right)
$$

of the matrix $A_{i-1-1}$. For each positive integer $k$ and each $S_{k} \in C_{k}(T)$ with $S_{k}=T_{r_{k}} \beta_{k}$, it follows by induction that the matrix $\left[\beta_{k}\right]$ is of the form

$$
\left[\begin{array}{ccccc}
B_{11} & & & & \\
B_{21} & B_{22} & & & 0 \\
B_{31} & B_{32} & B_{33} & & \\
& \ldots & & \ddots & \\
B_{m 1} & \ldots & B_{m m-1} & B_{m m}
\end{array}\right]
$$

where $B_{i j}, i, j=1,2, \ldots, m$, are matrices of order $\left(l_{i}-l_{i-1}\right) \times\left(l_{j}-l_{j-1}\right)$. Because, if $k=1$, then $\beta_{1} \beta=\beta \beta_{1}$. Since the spectrum of $T=T_{r} \beta$ is quasi-discrete, the matrix $\left[\beta_{1}\right]$ is of the form (3). Let $\left[\beta_{k}\right]$ be of the form (3). Then the rank of the group $O(T)$ $\cap G(T)_{i}$ is equal to the rank of the group $O\left(S_{k} T\right) \cap G\left(S_{k} T\right)_{i}$ since $V_{S_{k+1}} G(T)_{i}$ $=G\left(S_{k} T\right)_{i}$ for $i=1,2, \ldots, m$. Thus we see that the matrix $\left[\beta_{k} \beta\right]$ is of the form (2). Since $\beta_{k+1} \beta=\beta_{k} \beta \beta_{k+1}$, the matrix $\left[\beta_{k+1}\right]$ is of the form (3). We show that $C_{m+1}(T)$ $=C_{m+2}(T)$. If $S_{m+2} \in C_{m+2}(T)$ with $S_{m+2}=T_{r_{m+2}} \beta_{m+2}$, then we have $S_{m+2} T S_{m+2}^{-1}$ $=S_{m+1} T$ where $S_{m+1} \in C_{m+1}(T)$ with $S_{m+1}=T_{r_{m+1}} \beta_{m+1}$. From the equation above, we have $\beta_{m+2} \beta=\beta_{m+1} \beta \beta_{m+2}$. Since the matrix $\left[\beta_{m+1} \beta\right]$ is of the form (2), the mat$\operatorname{rix}\left[\beta_{m+1}\right]$ is also of the form (2). For the affine transformation $S_{m+1}=T_{r_{m+1}} \beta_{m+1}$, $S_{m+1} T S_{m+1}^{-1}=S_{m} T$ where $S_{m} \in C_{m}(T)$ with $S_{m}=T_{r_{m}} \beta_{m}$. Therefore $\beta_{m+1} \beta=\beta_{m} \beta \beta_{m+1}$ and

$$
r_{m+1}+\beta_{m+1}(r)=r_{m}+\beta_{m}(r)+\beta_{m} \beta\left(r_{m+1}\right) .
$$

Since $\left[\beta_{m}\right]$ and $\left[\beta_{m+1}\right]$ are of the form (2) and $\left[\beta_{m+1}\right]=\left[\beta_{m} \beta \beta_{m+1}\right]$, it follows that the blocks of $\left[\beta_{m}\right], A_{i-1}, i=2,3, \ldots, m$, are zero matrices. From (4) and the form of the matrix $\left[\beta_{m}\right]$ obtained above, we see that $T_{r_{m}}$ is of the form

$$
r_{m}=\left[\begin{array}{l}
0 \\
\vdots \\
0 \\
r_{m l_{1}+1} \\
\vdots \\
r_{m n}
\end{array}\right] .
$$

Continuing the argument $m$ times, for the affine transformation $S_{2}=T_{r_{2}} \beta_{2}$ belonging to $C_{2}(T)$, there exists an affine transformation $S_{1} \in C_{1}(T)$ such that $S_{2} T S_{2}^{-1} T^{-1}=S_{1}$, 
but it follows that $S_{1}$ is the identity map since the continuous group automorphism $\beta_{2}$ is the identity map and $r_{2}=\left(0, \ldots, 0, r_{2 l_{m-1}+1}, \ldots, r_{2 n}\right)$. Therefore $S_{1} \in C_{0}(T)$ and $S_{m+2} \in C_{m+1}(T)$. Consequently, $C_{m+1}(T)=C_{m+2}(T)$. Since the blocks $A_{i-1}$, $i=2,3, \ldots, m$, of the matrix $[\beta]$ are nonzero and moreover the first row in $A_{i-1-1}$, $\left(a_{l_{i-1}^{\prime}+1 l_{i-2}+1}, a_{l_{i-1}+1 l_{i-2}+2}, \ldots, a_{l_{i-1}+1 l_{i-1}}\right)$, is a nonzero vector. By the same manner used in Theorem 3.1, we can construct an affine transformation $S=T_{d} \beta^{\prime}$ such that $S \in C_{m+1}(T)$, but $S \notin C_{m}(T)$. Therefore $C N(T)=m+1$.

THEOREM 3.5. Let $T$ be an affine transformation of $X_{n}$ and let $\left(X_{n}, T\right)$ be a totally minimal dynamical system (with quasi-discrete spectrum) with $G N(T)=m$. Then $C_{m+1}(T)$ is a subgroup of the group consisting of all homeomorphisms of $X_{n}$.

Proof. As in Theorems 3.1 and 3.4, we may suppose that the affine transformation $T=T_{r} \beta$ is written as follows

$$
\begin{array}{r}
T:\left(x_{1}, x_{2}, \ldots, x_{n}\right) \rightarrow\left(x_{1}+r_{1}, \ldots, x_{l_{1}}+r_{l_{1}}, x_{l_{1}+1}\right. \\
+\sum_{j=1}^{l_{1}} a_{l_{1}+1 j} x_{j}+r_{l_{1}+1}, \ldots, x_{l_{2}} \\
+\sum_{j=1}^{l_{1}} a_{l_{2} j} x_{j}+r_{l_{2}}, \ldots, x_{l_{m-1}+1} \\
\left.+\sum_{j=1}^{l_{m-1}} a_{l_{m-1}+1 j} x_{j}+r_{l_{m-1}+1}, \ldots, x_{l_{m}}+\sum_{j=1}^{l_{m-1}} a_{l_{j} j} x_{j}+r_{l_{m}}\right) \\
\text { (additions mod 1), }
\end{array}
$$

where each $a_{i j}$ is an integer and indices $l_{j}, j=1,2, \ldots, m$, are integers such that $l_{m}=n$ and there is at the least one nonzero integer in

$$
\left\{a_{l_{j-1}+1 l_{j-2}+1}, a_{l_{-1}+1 l_{j-2}+2}, \ldots, a_{l_{j-1}+1 l_{j-1}}\right\} \quad \text { (but } l_{0}=0 \text { ). }
$$

Since $\left(X_{n}, T\right)$ is (totally) minimal, real numbers $r_{k}, k=1,2, \ldots, l_{1}$ are integrally independent. We consider affine transformations $Q_{k}=T_{r_{k}} \beta_{k}, k=0,1,2, \ldots, m$, where $T_{r_{k}}$ are translations of $X_{n}$ and $\beta_{k}$ a continuous group automorphism of $X_{n}$. Suppose now that the matrix $\left[\beta_{m}\right]$ is of the form

$$
\left[\begin{array}{ccccccc}
A_{11} & & & & & \\
A_{21} & A_{22} & & & 0 & \\
A_{31} & A_{32} & A_{33} & & & \\
& \cdots & & \ddots & & \\
A_{m 1} & & \ldots & & A_{m m-1} & A_{m m}
\end{array}\right]
$$

where $A_{i j}, i, j=1,2, \ldots, m$, are matrices of order $\left(l_{i}-l_{i-1}\right) \times\left(l_{j}-l_{j-1}\right)$ (but $l_{0}=0$ ). The form of $[\beta]$ has the form (1) and in particular $A_{i i}, i=1,2, \ldots, m$, are the identity matrices. We put

$$
Q_{m-j} T Q_{m-j}^{-1} T^{-1}=Q_{m-1-j}, \quad j=0,1,2, \ldots, m-1 .
$$


From the relation (2), the matrices $\left[\beta_{m-j}\right], j=1,2, \ldots, m-1$, are of the form (1). Moreover, from $\left[\beta_{m-j} \beta\right]=\left[\beta_{m-1-j} \beta \beta_{m-j}\right],\left[\beta_{m-j}\right], j=1,2, \ldots, m$, are the matrices such that

$$
\left[\begin{array}{cccccccc}
E_{11} & & & & & & \\
& \ddots & & 0 & & & \\
& 0 & & & & & \\
B_{j+2} & 0 & \cdots & 0 & E_{j+2 j+2} & & \\
\cdots & \ddots & \cdots & & \ddots & & \\
B_{m 1} & \cdots & B_{m m-1-j} & 0 & \cdots & 0 & E_{m m}
\end{array}\right]
$$

where each $E_{i i}$ is the identity matrix of order $\left(l_{i}-l_{i-1}\right) \times\left(l_{i}-l_{i-1}\right)$ and each $B_{i j}$ is the matrix of order $\left(l_{i}-l_{i-1}\right) \times\left(l_{j}-l_{j-1}\right)$ (but $\left.l_{0}=0\right)$. From this fact and the relation (2), the translation $T_{r_{m-}}$ is of the form

$$
r_{m-j}=\left[\begin{array}{l}
0 \\
\vdots \\
0 \\
r_{m-j l_{j-1}+1} \\
\vdots \\
r_{m-j n}
\end{array}\right], \quad j=2,3, \ldots, m-1
$$

Thus it follows that if an affine transformation $S$ is a transformation consisting of a translation composed with continuous group automorphisms $\beta^{\prime}$ of $X_{n}$ such that the matrix $\left[\beta^{\prime}\right]$ is of the form (1), then $S \in C_{m+1}(T)$. Here we show that $C_{m+1}(T)$ is a subgroup. For $S, S^{\prime \prime} \in C_{m+1}(T)$ with $S=T_{r}, \beta^{\prime}$ and $S^{\prime \prime}=T_{r^{\prime \prime}} \beta^{\prime \prime}$. Let us put

$$
D=\left(S^{\prime \prime} S^{-1}\right) T\left(S^{\prime \prime} S^{-1}\right)^{-1} T^{-1},
$$

then $\beta^{(3)}\left(=\left(\beta^{\prime \prime} \beta^{\prime-1}\right) \beta\left(\beta^{\prime \prime} \beta^{\prime-1}\right)^{-1} \beta^{-1}\right)$ is a continuous group automorphism of $X_{n}$ and $T_{r}(3)$ a translation of $X_{n}$ where

$$
\begin{aligned}
r^{(3)}= & r^{\prime \prime}+\beta^{\prime \prime} \beta^{\prime-1}\left(r-r^{\prime}\right)-\beta^{\prime \prime} \beta^{\prime-1} \beta \beta^{\prime} \beta^{\prime \prime-1}\left(r^{\prime \prime}\right) \\
& -\beta^{\prime \prime} \beta^{\prime-1} \beta\left(r^{\prime}\right)-\beta^{\prime \prime} \beta^{\prime-1} \beta \beta^{\prime} \beta^{\prime \prime}-1 \beta^{-1}(r) .
\end{aligned}
$$

The affine transformation $D=T_{r^{(3)} \beta^{(3)}}$ belongs to $C_{m+2}(T)$, because the matrices $\left[\beta^{\prime}\right]$ and $\left[\beta^{\prime \prime}\right]$ are of the form (1). Consequently $S^{\prime \prime} S^{-1} \in C_{m+1}(T)$ since

$$
D=\left(S^{\prime \prime} S^{-1}\right) T\left(S^{\prime \prime} S^{-1}\right)^{-1} T^{-1} \text { and } C N(T)=m+1 .
$$

We have shown that $C_{m+1}(T)$ is a subgroup.

As before, $(\Omega, \Sigma, \mu)$ is a finite measure space and $T$ is a totally ergodic automorphism of $(\Omega, \Sigma, \mu)$ with quasi-discrete spectrum. We consider a normalized measure space $(\Omega, \Sigma, \mu)$.

In order to prove the following lemma, we invoke properties of entropy. 
Lemma 3.6. Let $S$ and $W$ be as in Corollary 2.6 and let $T^{\prime}$ be a metric automorphism induced by $T$ such that $T^{\prime}=S W$. If for any $f \in O_{\mu}(T), Y$ is a subgroup generated by an orbit of $f$ under $V_{S}$, then $Y$ is finitely generated.

Proof. We denote by $G$ the subgroup of $Y$ generated by the set

$$
\left\{V_{S}^{j} f: j=1,2, \ldots\right\} .
$$

If $V_{S} G \neq G$. Then it is well known that $T^{\prime}=S W$ has positive entropy. On the other hand, since $T$ is totally ergodic and has quasi-discrete spectrum, the entropy of $T$ is zero. Thus $V_{S} G=G$ and

$$
f=V_{S}^{n_{1}} f^{q_{1}} \cdot V_{S^{2}}^{n_{2}} f^{q_{2}} \ldots \ldots V_{S}^{n_{k}} f^{q_{k}}
$$

where each $n_{j}$ is an integer and each $q_{j}$ an integer. If $G^{\prime}$ is a subgroup generated by $\left\{V_{S}^{j} f: j=1,2, \ldots, k\right\}$ where $k=\max \left\{n_{1}, n_{2}, \ldots, n_{k}\right\}$, it follows that $V_{S} G^{\prime}=G^{\prime}$. Thus $Y=G^{\prime}$ and $Y$ is finitely generated.

We denote by $T^{\prime}$ the metric automorphism induced by an automorphism $T$.

THEOREM 3.7. If $G_{\mu} N(T)=m$, then $C N\left(T^{\prime}\right)=m+1$. Furthermore, if $G_{\mu} N(T)=+\infty$ then $C N\left(T^{\prime}\right)=+\infty$.

Proof. Since $T$ is totally ergodic and has quasi-discrete spectrum, $O_{\mu}(T)$ is an orthonormal base of $L^{2}\left(\Sigma(\mu)\right.$ ), and, by Corollary 2.6, the metric automorphism $T^{\prime}$ has a representation $T^{\prime}=S W$ on $\Sigma(\mu)$ for metric automorphisms $S$ and $W$ such that $V_{S} O_{\mu}(T)=O_{\mu}(T)$ and $W$ has each function in $O_{\mu}(T)$ as a proper function. For any $f \in O_{\mu}(T)$, it follows from Lemma 3.6 that $Y(f)$, the smallest subgroup generated by an orbit of $f$ under $V_{S}$, is finitely generated. Since $T$ is totally ergodic $Y(f)$ is torsion free. Here we suppose that the number of generators of $Y(f)$ is $n$. Since there exists a nontrivial $T$-invariant sub $\sigma$-algebra $\Phi(\mu)$ such that

$$
L^{2}(\Phi(\mu))=\overline{\operatorname{span} Y(f)}
$$

and $T^{\prime}$ has quasi-discrete spectrum on $L^{2}(\Phi(\mu))$, it follows from the proof of Theorem 1.2 that there exists the $n$-dimensional torus $X_{n}$ such that the dynamical system $\left(X_{n}, A_{f}\right)$ is totally minimal, and that $T^{\prime}$ restricted to $\Phi(\mu)$ is isomorphic to the metric automorphism $A_{f}^{\prime}$ induced by $A_{f}$; in other words, $\varphi_{f} T^{\prime}=A_{f}^{\prime} \varphi_{f}$ where $\varphi_{f}$ is a metric isomorphism from the measure algebra $\Phi(\mu)$ to the measure algebra associated with the measure space consisting of the Borel field of $X_{n}$ and the normalized Haar measure. We show first that $C N\left(T^{\prime}\right)=m+1$ if $G N(T)=m$ and $m$ is an integer. Suppose now that $G N\left(A_{f}\right) \leqq G_{\mu} N(T)-1(=m-1)$ for each $f \in O_{\mu}(T)$. Then we have

and

$$
G_{\mu}(T) \cap Y(f)=G_{\mu}(T)_{m-1} \cap Y(f)
$$

$$
\begin{aligned}
G_{\mu}(T) & =\bigcup\left\{G_{\mu}(T) \cap(K \times Y(f)): f \in O_{\mu}(T)\right\} \\
& =\bigcup\left\{G_{\mu}(T)_{m-1} \cap(K \times Y(f)): f \in O_{\mu}(T)\right\} \\
& =G_{\mu}(T)_{m-1} .
\end{aligned}
$$


This contradicts $G_{\mu} N(T)=m$. Therefore there exists a function $f \in O_{\mu}(T)$ such that $G N\left(A_{f}\right)=m$. Let us put for each $f \in O_{\mu}(T)$

$$
C_{0}\left(A_{f}\right)=\left\{A: A^{\prime}=\varphi_{f} S^{\prime} \varphi_{f}^{-1} \text { for } S^{\prime} \in C_{0}\left(T^{\prime}\right)\right\}
$$

and

$$
C_{n}\left(A_{f}\right)=\left\{A: A^{\prime}=\varphi_{f} S^{\prime} \varphi_{f}^{-1} \text { for } S^{\prime} \in C_{n}\left(T^{\prime}\right)\right\}, \quad n=1,2, \ldots
$$

$C_{n}\left(A_{f}\right), n=0,1,2, \ldots$, are the generalized commuting classes of affine transformations with respect to the affine transformation $A_{f}$. It follows from Theorem 3.4 that for $f \in O_{\mu}(T)$ with $G N\left(A_{f}\right)=m, C N\left(A_{f}\right)=m+1$. It is clear that $C N\left(A_{g}\right) \leqq m$ for $g \in O_{\mu}(T)$ with $G N\left(A_{g}\right) \leqq m-1$. Consequently we see that

$$
m+1=\max \left\{n: C_{n}\left(A_{f}\right)=C_{n+1}\left(A_{f}\right) \text { for } f \in O_{\mu}(T)\right\} .
$$

Therefore we have $C N\left(T^{\prime}\right)=m+1$. It remains to show that if $G_{\mu} N(T)=\infty$ then $C N\left(T^{\prime}\right)=\infty$. For the finitely generated group $Y(f)$ of a member $f$ of $O_{\mu}\left(T^{\prime}\right)$, we denote by $g_{0}$ a function $g$ such that $g \in O_{\mu}(T)$ and $g \notin Y(f)$, and by $g_{1}$ a function $g$ such that $g \in O_{\mu}(T)$ and $g \notin Y(f) \cup Y\left(g_{0}\right)$ and so on. Then we can choose infinitely many set functions $\left\{g_{j}: j=0,1,2, \ldots\right\}$ such that $g_{j}$ belongs to a group of distinct order for $j=0,1,2, \ldots$. Here we put

$$
G_{l_{m}}=\prod_{j=0}^{m} Y\left(g_{j}\right), \quad m=0,1,2, \ldots
$$

and let the index $l_{m}$ be the least integer such that $\left(K \times \prod_{j=0}^{m} Y\left(g_{j}\right)\right) \cap G_{\mu}(T)$ $\subset G_{\mu}(T)_{l_{m}}$. Then $V_{T} G_{l_{m}}=G_{l_{m}}$ and $l_{k} \uparrow+\infty$ as $k \rightarrow+\infty$. Let $X$ be the dual space of a discrete group $G_{l_{m}}$ and $Q$ a transformation on $X$ induced by $T$. Then $Q$ is totally ergodic with respect to Haar measure $\mu^{\prime}$ on $X$ and has quasi-discrete spectrum and $G_{\mu}, N(Q)=l_{m}$. We see from Theorem 3.4 that the generalized commuting order of $Q$ is $l_{m}+1$. From this, we follow that $C N\left(T^{\prime}\right) \geqq l_{m}+1$. Since $m$ is an arbitrary positive integer, we have $C N\left(T^{\prime}\right)=\infty$.

THEOREM 3.8. If $C N\left(T^{\prime}\right)=m$, then $G_{\mu} N(T)=m-1$. Furthermore, if $C N\left(T^{\prime}\right)=+\infty$ then $G_{\mu} N(T)=+\infty$.

The proof is an application of Theorems 3.1, 3.4 and 3.7.

COROLlaRY 3.9. If $G_{\mu} N(T)=m$, then $C_{m+1}\left(T^{\prime}\right)$ is a subgroup of the group consisting of all metric automorphisms of $\Sigma(\mu)$ onto itself. Furthermore, if $G_{\mu} N(T)=$ $+\infty$ then $C_{\infty}\left(T^{\prime}\right)=\bigcup_{n=0}^{\infty} C_{n}\left(T^{\prime}\right)$ is a subgroup.

This corollary is proved by Theorems 3.5 and 3.7.

\section{REFERENCES}

1. L. M. Abramov, Metric automorphisms with quasi-discrete spectrum, Izv. Akad. Nauk SSSR Ser. Mat. 26 (1962), 513-530; English transl., Amer. Math. Soc. Transl. (2) 39 (1964), 37-56. MR 26 \#606. 
2. R. L. Adler, Generalized commuting properties of measure-preserving transformations, Trans. Amer. Math. Soc. 115 (1965), 1-13. MR 34 \#2828.

3. H. Anzai, Ergodic skew product transformations on the torus, Osaka Math. J. 3 (1951), 83-99. MR 12, 719.

4. N. Aoki, On generalized commuting properties of metric automorphisms. I, II, Proc. Japan Acad. 44 (1968), 467-471; 45 (1969), 17-19.

5. - On zero entropy and quasi-discrete spectrum for automorphisms, Proc. Japan Acad. 45 (1969), 20-24.

6. R. R. Bahadur, Measurable subspaces and subalgebras, Proc. Amer. Math. Soc. 6 (1955), 565-570. MR 17, 286.

7. R. Ellis, Distal transformation groups, Pacific J. Math. 8 (1958), 401-405. MR 21 \#96.

8. H. Furstenberg, Strict ergodicity and transformation of the torus, Amer. J. Math. 83 (1961), 573-601. MR 24 \#A3263.

9. L. Gillman and M. Jerison, Rings of continuous functions, The University Series in Higher Math., Van Nostrand, Princeton, N. J., 1960. MR 22 \#6994.

10. F. Hahn, On affine transformations of compact abelian groups, Amer. J. Math. 85 (1963), 428-446. MR 27 \#5889.

11. F. Hahn and W. Parry, Minimal dynamical systems with quasi-discrete spectrum, J. London Math. Soc. 40 (1965), 309-323. MR 30 \#5292.

12. P. R. Halmos and J. von Neumann, Operator methods in classical mechanics. II, Ann of Math. (2) 43 (1942), 332-350. MR 4, 14.

13. P. R. Halmos and H. Samelson, On monothetic groups, Proc. Nat. Acad. Sci. U.S.A., 28 (1942), 254-258. MR 4, 2.

14. A. H. M. Hoare and W. Parry, Affine transformations with quasi-discrete spectrum. I, J. London Math. Soc. 41 (1966), 88-96, MR 32 \#4207.

15. - Affine transformations with quasi-discrete spectrum. II, J. London Math. Soc. 41 (1966), 529-530. MR 33 \#2797.

16. W. Parry, Entropy and generators in ergodic theory, Benjamin, New York, 1969.

17. L. Pontrjagin, Topological groups, GITTL, Moscow, 1938; English transl., Princeton Math. Series, vol. 2, Princeton Univ. Press, Princeton, N. J., 1939; 5th printing, 1958. MR 1, 44; MR 19, 867.

JOSAI UNIVERSITY,

Sakado, Saitama, Japan 